solution versus base as an adjunct to oral griseofulvin in patients with onychomycosis. Clin Exp Dermatol 1987;12:175-7.

8 Petranyi G, Ryder NS. Stutz A Allylamine derivatives: new class of syntheric antifungal agents inhibiting fungal squalene epoxidase. Science 1984,224: antifungal agents inhibiting fungal squalene ep

Stutz A. Synthesis and structure-activity correlations within allylamine antimycotics. Ann N Y Acad Sci 1988;544:46-62.

10 Van de Bossche H, Willemsens G, Cools W, Lauwers WFJ, Lejeune L. Biochemical effects of miconazole on fungi. Inhibition of ergosterol biosynthesis in Candida albicans. Chemical and Biological Interaction 1978;21:59-78.

11 Back DJ, Stevenson P, Tjia JF. Comparative effects of two antimycotic agents, ketoconazole and terbinafine on the metabolism of tolbutamide, ethinyloestradiol, cyclosporin and ethoxycoumarin by human liver microsomes in vitro. Brf Clin Pharmacol 1989;28: 166-70.

12 Savin R. Successful treatment of chronic tinea pedis (mocassin type) with terbinafine (Lamisil). Clin Exp Dermatol 1989;14:116-9.
13 Villars V, Jones TC. Present status of the efficacy and tolerability of terbinafine (Lamisil) used systemically in the treatment of dermatomycoses of skin and nails. Fournal of Dermatological Treatment 1990;1(suppl 2):33-9.

14 Goodfield MJD, Rowell NR, Forster RA, Evans EGV, Raven A. Treatmen of of dermatophyte infection of the finger and toenails with terbinafin (SF 86-327, Lamisil), an orally active fungicidal agent. Br f Dermato

15 Zaias N, Serrano L. The successful treatment of finger Trichophyton rubrum onychomycosis with oral terbinafine. Clin Exp Dermatol 1989;14:120-3.

16 Zaias N, Drackman DA. A method for the determination of drug effectiveness in onychomycosis. I Am Acad Dermatol 1983;9:912-9.

17 Harland CC, Goodfield MJD, Evans EGV. A follow up study of terbinafine (SF-327, Lamisil) in the treatment of onychomycosis. Br $\mathrm{F}$ Dermato 1990;123(suppl 37):31.

(Accepted 24 February 1992)

\title{
Delayed diagnosis in non-insulin dependent diabetes mellitus
}

\author{
B M Singh, D M A Jackson, R Wills, J Davies, \\ P H Wise
}

\section{Department of \\ Endocrinology, Charing \\ Cross Hospital, London W6 8RF \\ B M Singh, senior registra \\ D M A Jackson, registrar \\ $\mathrm{R}$ Wills, sister, \\ diabetes care \\ J Davies, clinical \\ nurse specialist, diabetes \\ $\mathrm{P} \mathrm{H}$ Wise, consultant \\ endocrinologist}

Correspondence to: Dr Singh.

BMF 1992;304:1154-5
Disease duration is a recognised factor in the development of microvascular complications in non-insulin dependent diabetes, ' and delayed presentation might contribute to the prevalence of such complications at diagnosis. ${ }^{23}$ This study aims to examine the potential for achieving earlier diagnosis of non-insulin dependent diabetes.

\section{Methods and results}

We studied 100 newly diagnosed patients ${ }^{4}(36$ men, 54 women; mean age 60 (SD 11) years) presenting consecutively to the diabetes day centre at Charing Cross Hospital. The diagnosis had been made by general practitioners in 54 patients, by hospital departments in 33, during independent medical examinations in eight, and by the patients themselves in five. Patients were interviewed by standardised questionnaire to determine the nature and duration of their symptoms at presentation. Symptoms evaluated were thirst, polyuria, lethargy, and weight loss - the symptoms of diabetes most commonly known to nondiabetic people. ${ }^{5}$ The nature of contacts with doctors after symptoms had been recognised was recorded. Each patient marked the presence and duration of symptoms listed on a standard form to document those they had not previously volunteered.

A major symptom of diabetes had been reported to referring doctors by 39 patients. Nine patients had other specific or atypical presentations: four with infection (balanitis (2); abscess; necrotising fasciitis); four with visual disturbance (central retinal artery or vein occlusion (3); diabetic retinopathy); one with diabetic amyotrophy. The diagnosis was made in 31 patients presenting with symptoms not related to diabetes, and 21 were found at routine screening. At their first visit to the diabetes day centre 57 patients reported one or more of the major symptoms: 24 reported one symptom; 18, two; 14, three; and one reported having all four. Forty three patients still claimed to be asymptomatic (table). No individual symptom was widely volunteered, and thirst and polyuria in combination were recognised by only 17 patients. After completing the symptom inquiry form, 80 patients recognised the presence of one or more further symptoms: 35 , one; 26 , two; 17 , three; and 2 recognised four. Thirteen patients identified no further symptoms, but only seven continued to be truly asymptomatic (table). Taking reports of previously recognised and unrecognised symptoms together, all four symptoms were commonly reported (table).
Eighteen patients now recognised one major symptom; 14, two; 40, three; and 21, four. Fifty eight patients recognised thirst and polyuria in combination, with only 19 denying either. Overall, 39 patients had had symptoms for more than 12 months.

Numbers of newly presenting patients with non-insulin dependent diabetes $(n=100)$ categorised according to duration of previously recognised or unrecognised symptoms

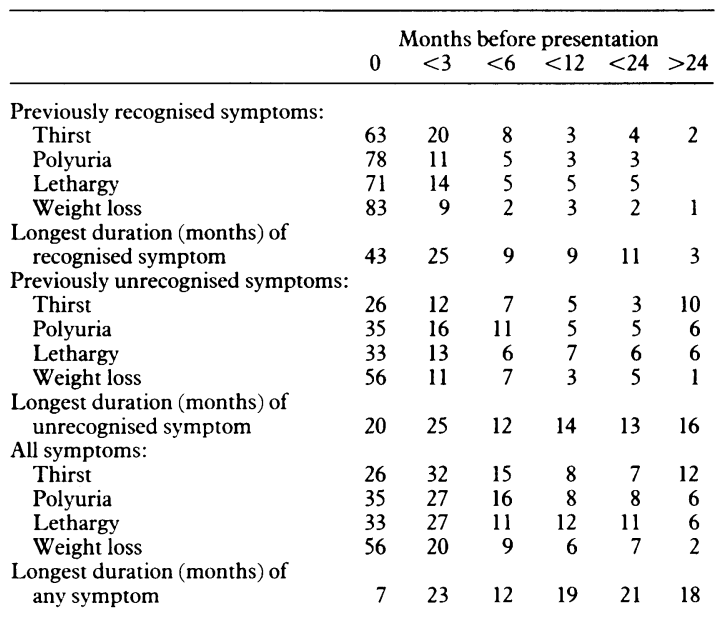

Seventy seven patients had seen a doctor while they had the recognised symptoms of diabetes. In 42 there had been no reason to suspect a diagnosis of diabetes. In eight patients the diagnosis had been made a median of 13 (range 3-60) months previously, with findings of raised random blood glucose'concentration in eight and glycosuria in seven, but the patients had not been informed. Seven of these patients had been seen in hospital departments. In 21 patients the diagnosis had been missed 6 (1-120) months before presentation. Of these, 12 had attended their general practice ( 3 with recurrent vaginal candidiasis, 1 with recurrent cellulitis, 8 with osmotic symptoms) and nine attended hospital departments ( 4 in ophthalmology ( 3 cataracts, 1 glaucoma), 2 in surgery ( 1 abscess, 1 "prostatism"), 2 in casualty (diabetic amyotrophy, recurrent abscess) and 1 in psychiatry). Urine had been tested in only one of these patients. Four patients had been informed of the diagnosis but failed to have further follow up for periods of 6-12 months before presentation. Thus, among 33 patients $(43 \%$ of those who had seen a doctor) the potential for treatment had existed 9 (1-120) months previously and occurred at hospital departments in 16 patients, in general practice in 13 , and after independent medical examinations in four.

\section{Comment}

Our data show that asymptomatic non-insulin dependent diabetes is unusual. Patients often have symptoms but do not recognise them as being abnormal. Almost 
$40 \%$ of patients had symptoms for more than 12 months before starting treatment. Our previous study of the general public's knowledge of diabetes showed that fewer than one in 20 could name the combination of thirst and polyuria as symptoms, suggesting that poor levels of public health education for symptoms related to diabetes may have been an important cause of late presentation. ${ }^{5}$ Furthermore, earlier treatment might have been started in a third of our patients in whom the diagnosis either was made but not acted on or was overlooked. The results of this audit emphasise not only the need to raise awareness of diabetes among health professionals but also to increase the knowledge of major symptoms of diabetes by health promotion among the general public.

1 Knuiman MW, Welborn TA, McCann VJ, Stanton KG, Constable IJ. Prevalence of diabetic complications in relationship to risk factors Diabetes 1986;35:1332-9.

2 Hillson RA, Hockaday TDR, Newton DJ, Pim B. Delayed diagnosis of noninsulin dependent diabetes is associated with greater metabolic and clinical abnormality. Diabetic Med 1985;2:383-6.

3 Mincu I. Micro and macroangiopathies and other chronic degenerative complications in newly diagnosed diabetes mellitus. Medicine International 1970;304:1331-4.

4 WHO Expert Committee on Diabetes Mellitus. WHO Tech Rep Ser 1990;646.

5 Jackson DMA, Wills R, Davies J, Meadows K, Singh BM, Wise PH. Public awareness of the symptoms of diabetes mellitus Diabetic Med 1991;8:971-2.

(Accepted 3 fanuary 1992)

\title{
Maternal serum anti-D antibody concentration and assessment of rhesus isoimmunisation
}

\author{
K H Nicolaides, C H Rodeck
}

Harris Birthright Research King's College School of Medicine and Dentistry, London SE5 8RX K H Nicolaides, director

Department of Obstetrics and Gynaecology, University College and Middlesex School of Medicine, London C H Rodeck, professor

Correspondence to: Dr Nicolaides:

BMF 1992;304:1155-6 Centre for Fetal Medicine,

The association between maternal serum haemolytic antibody concentration and outcome of pregnancy has been recognised from the 1950s and measurement of maternal anti-D antibody has been the traditional method of managing pregnancies in which red blood cell isoimmunisation has occurred. Fetal blood sampling from an umbilical cord vessel allows direct assessment of the severity of the disease by measurement of the haemoglobin concentration. ${ }^{1}$ The aim of this study was to determine the association between maternal serum anti-D antibody concentration and fetal anaemia.

\section{Patients, methods, and results}

Fetal blood was obtained by fetoscopy ${ }^{2}(n=93)$ or cordocentesis $^{3}(n=144)$ from 237 pregnancies in which rhesus isoimmunisation had occurred at 17-38 weeks' gestation. The study was cross sectional, and the data were derived from fetuses that had not yet received transfusions. The direct Coombs test was positive in

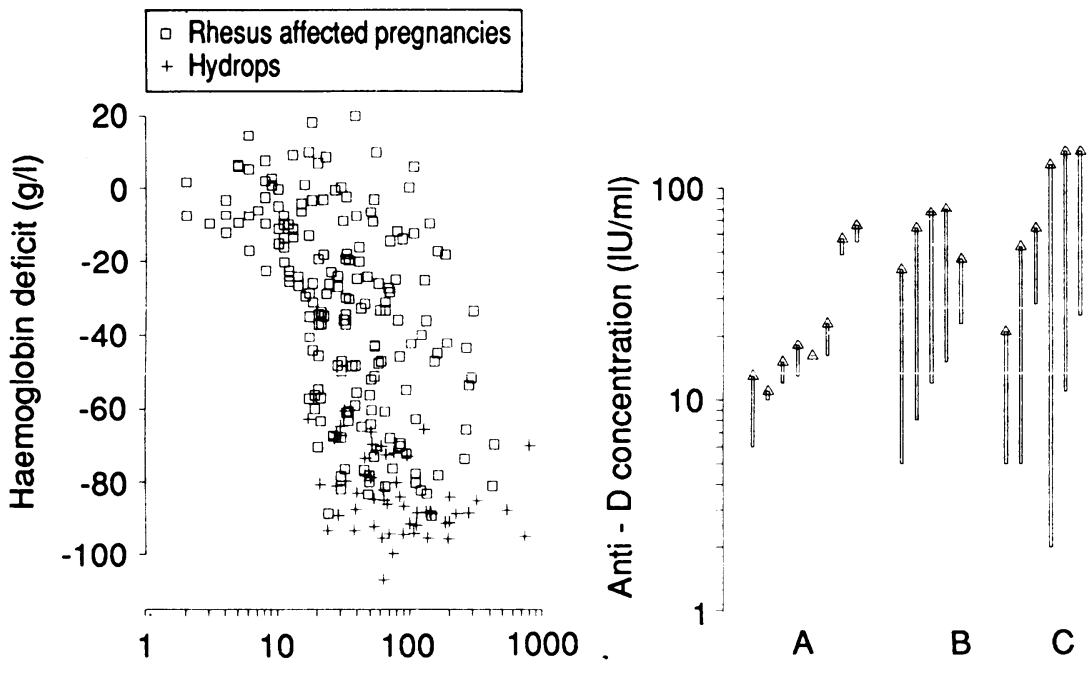

Anti - D concentration (IU/ml)

Left: maternal serum anti-D concentration $(I U / \mathrm{ml})$ and fetal haemoglobin concentration deficit (reduction in haemoglobin $(\mathrm{g} / \mathrm{l})$ from normal mean for gestation) in 237 fetuses from rhesus affected pregnancies. Fifty six hydropic fetuses were severely anaemic (deficit $>60 \mathrm{~g} / \mathrm{l}$ ). When maternal anti-D concentration was $\leqslant 15 \mathrm{IU} / \mathrm{ml}$ fetuses were only mildly anaemic (deficit $<30 \mathrm{~g} / \mathrm{l}$ ). Right: increase in maternal anti-D concentration after first cordocentesis in 19 non-anaemic fetuses; with increase $\leqslant 15 \mathrm{IU} / \mathrm{ml}$ fetuses did not develop significant anaemia $(A)$ whereas with increase $>15 \mathrm{IU} / \mathrm{ml}(B$ and $C)$ six fetuses developed fetal anaemia $(C)$ all fetuses, and Kleihauer staining showed that all samples contained only fetal red cells. The fetal haemoglobin concentration was determined (Coulter S Plus counter, Coulter Electronics, Luton) and the haemoglobin deficit (the amount by which the values differed from the normal mean for gestation) was calculated (normal haemoglobin $=85 \pm 1.4$ gestational weeks, residual $\mathrm{SD}=10 \mathrm{~g} / \mathrm{l}$; this was established from the study of 726 pregnancies with cordocentesis for various indications in which the fetus was retrospectively found to be normal). ${ }^{1}$ The maternal serum anti-D antibody concentration was measured in samples obtained by venepuncture immediately before fetal blood sampling with an autoanalyser calibrated against the British anti-D working standard 72/229 (Technicon Corporation) ${ }^{4}$; the intra-assay coefficient of variation was $10 \%$.

Although $\log _{10}$ maternal anti-D antibody concentration and fetal haemoglobin deficit were significantly associated (figure; $r=0.57, \mathrm{n}=237, \mathrm{p}<0.0001$ ), the wide scatter of results prevents useful clinical application. Nevertheless, in all 42 pregnancies with a maternal anti-D concentration $\leqslant 15 \mathrm{IU} / \mathrm{ml}$ the fetuses were at most mildly anaemic (haemoglobin deficit $<30 \mathrm{~g} / \mathrm{l})$.

In 19 pregnancies in which the fetus was not anaemic further maternal anti-D measurement and cordocentesis were performed two to three weeks subsequently. In the eight pregnancies in which the increase in maternal anti-D antibody was $\leqslant 15 \mathrm{IU} / \mathrm{ml}$ the fetuses remained at most mildly anaemic; in contrast, in six of the 11 pregnancies with an increase in maternal anti-D antibody $>15 \mathrm{IU} / \mathrm{ml}$ the fetus became moderately or severely anaemic (figure).

\section{Comment}

The significant association between the degree of fetal anaemia and maternal serum anti-D antibody concentration is compatible with the pathophysiology of rhesus disease, which involves the extravascular destruction of fetal red cells that are coated with transplacentally derived maternal anti-D antibody. In addition to intra-assay variation, the wide scatter of results may be due to individual variation in rate of transfer of antibody across the placenta, expression of antigens in fetal red cells, and fetal erythropoiesis in response to haemolysis.

In managing rhesus affected pregnancies cordocentesis gives accurate information on the severity of the disease and provides access for fetal intravascular blood transfusion. ${ }^{13}$ Even with experienced operators, however, the risk of fetal death from cordocentesis is about $1 \%$. Furthermore, the procedure is commonly associated with fetomaternal haemorrhage and worsening of the disease. ${ }^{5}$ If the maternal anti-D antibody concentration is $\leqslant 15 \mathrm{IU} / \mathrm{ml}$ the fetus is at most mildly anaemic, and therefore invasive antenatal intervention may not be indicated. In contrast, with a concentration 